\title{
Wie können wir medizinische Qualität im Krankenhaus managen?
}

„Qualität im Krankenhaus“ ist seit Jahren ein Themenkomplex, der viele Krankenhäuser, deren Mitarbeiter, Kostenträger, Verbände, die Politik und selbstverständlich auch die Patienten beschäftigt. Dabei wurden verschiedene Zertifizierungssysteme, Richtlinien, Leitlinien und neue Berufsbilder entwickelt, die sich originär mit dem wichtigen Punkt der „Qualität“ beschäftigen. Allerdings wird bei näherer Betrachtung immer wieder die Frage aufgeworfen, ob mit diesen Bemühungen, auch immer die medizinische Qualität, nämlich der Verbesserung der Behandlung abgebildet werden soll.

\section{Definition der , medizinischen Qualitåt" $\nabla$}

Da Qualität mit dem Synonym von „Güte“ gleichzusetzen ist, kann medizinische Qualität lediglich auf eine gute medizinische Leistung abzielen, die sich an den Ergebnissen der Patientenbehandlung messen lässt.

\section{Durchsetzbarkeit der „medizinischen Qualität* \\ $\checkmark$}

Damit eine Messung der Ergebnisse überhaupt möglich ist, müssen valide und verifizierbare Kriterien festgelegt werden, die eine medizinische Qualitätskontrolle möglich machen. Beispiele hierfür können die Häufigkeit von Behandlungsfehlern, Operationstyp, Sterblichkeit, etc. sein. Diese beispielhaft genannten, messbaren Kriterien stellen „harte“ Fakten dar, die u.a. unmittelbare Auswirkung auf das Wohl und Wehe des Patienten haben.

Eine Auswertung der medizinischen Qualität nach diesen Kriterien bedeutet auch, dass man anhand der Ergebnisse mit den Beteiligten an dem Behandlungsprozess in Diskussion getreten muss. Hier fängt in den meisten Fällen das größte Problem an, denn die Beteiligten werden möglicherweise mit Fehlern ihrer Behandlungsmethoden konfrontiert. Dadurch wird ein Tabuthema in der Krankenhauswelt aufgegriffen, das bislang eher nicht offen diskutiert wurde. Dieser Paradigmenwechsel erfordert eine Änderung der Unternehmenskultur, hin zu Transparenz und Vertrauen.

Dieses Vertrauen muss man allerdings bei diesem sensiblen Thema immer wieder neu gewinnen. Hierzu muss ein Qualitätsmanagementverfahren durchgeführt werden, welches einfach, nachvollziehbar und damit akzeptabel ist. Es darf dadurch kein Mehraufwand durch den Behand- ler entstehen, da grundsätzlich schon eine $\mathrm{Ab}$ neigung besteht, zusätzliche Daten zu erfassen (s. BQS, Erfassungsbögen Darm-, Brustzentren, etc.). Mithin muss auf den bestehenden Datenbestand zurückgegriffen werden, der bereits zur Verfügung steht.

Des Weiteren muss den Beteiligten klar gemacht werden, dass die Messung der Medizinischen Qualität dazu dienen soll, den Behandlungserfolg bei den Patienten zu verbessern. Es darf nicht der Eindruck entstehen, dass diese Form des Qualitätsmanagements als Begründung zu arbeitsrechtlichen Maßnahmen gegen die Beteiligten herhalten darf, soweit negative Ergebnisse eintreten. Die Vertrauensbasis wäre zerstört.

Um die Leistungen der Einzelnen vergleichen zu können, muss eine Gruppe von Fachabteilungen oder Kliniken gefunden werden, die miteinander vergleichbar sind. Durch das Benchmarking ist es besser möglich, die Stärken und Schwächen der Einzelnen darzustellen, die anschließend die Unterschiede herausarbeiten sollten, um sich weiter zu verbessern. Diese Vorgehensweise setzt wiederum Transparenz, Vertrauen und Wille zur Veränderung voraus, mithin eine Änderung der Unternehmenskultur.

Darüber hinaus ist es weiterhin notwendig, Anreize und Ziele zu setzen, um den Prozess zu noch besserer Qualität in Gang zu halten. Dies kann beispielsweise durch finanzielle Anreize im Arbeitsvertrag oder trägerweite abgestimmte Aktionen bestehen

Schließlich ist festzuhalten, dass medizinisches Qualitätsmanagement „Chefsache“ sein muss. Eine Delegation dieses wichtigen Themas ist nicht angezeigt.

\section{Fazit}

$\nabla$

Damit man medizinische Qualität managen kann, müssen nach Auffassung des Verfassers folgende Faktoren erfüllt sein:

Transparenz - Vertrauen - klarer, einfacher Datenbestand - offene Unternehmenskultur Benchmarking - Setzung von Anreizen - keine Delegation der Aufgabe

Autorenerklärung: Der Autor erklärt, dass kein finanzieller Interessenkonflikt in Bezug auf dieses Manuskript besteht.
S. John

Qualitätsmanagement

Schlüsselwörter

Medizinisches Qualitätsmanagement

- Krankenhaus

Benchmark

Key words

health care quality

hospital

benchmark

Institut

HELIOS Klinikum Wuppertal

Bibliografie

Dol 10.1055/s-0028-1085593 Dtsch Med Wochenschr 2008; 133: S149 - @ Georg Thieme Verlag KG Stuttgart - New York . ISSN 0012-0472

Korrespondenz RA Sascha John Geschäftsführer HELIOS Klinikum Wuppertal Heusnerstr. 40 D-42283 Wuppertal Tel. +49 202 896-2916 eMail sascha.john@ helios-kliniken.de 